Ophthalmologe 2005 -102:188-190

DOI 10.1007/s00347-003-0987-8

Online publiziert: 3. März 2004

(c) Springer Medizin Verlag 2004

R. Kiel ${ }^{1}$ A. Martinez ${ }^{2}$ M. E. Karlen ${ }^{2} \cdot$ T. Wolfensberger ${ }^{1} \cdot$ P. de Gottrau ${ }^{2}$

${ }^{1}$ Universitäts-Augenklinik Jules Gonin, Lausanne, Schweiz

${ }^{2}$ Augenklinik, Kantonsspital, Freiburg, Schweiz

\title{
Akute unilaterale Visusminderung
}

\section{Anamnese}

Ein 43-jähriger männlicher Patient wurde in unserer Notfallambulanz mit einer seit wenigen Stunden aufgetretenen schmerzlosen Visusreduktion am linken Auge ohne andere Allgemeinsymptomatik vorstellig. Die weitere Anamnese ergab keine Hinweise auf andere chronische Erkrankungen.

\section{Befund}

Der augenärztliche Status zeigte einen bestkorrigierten Visus von 0,4 am betroffenen linken Auge (LA). Die Sehschärfe am rechten Auge (RA) betrug 1,o s.c. Die vorderen Augenabschnitte waren beiderseits bis auf ein leichtes linksseitiges afferentes Pupil- lendefizit regelgerecht. Die Augenfundi zeigten eine leichte Tortuositas vasorum (RA=LA) mit einer temporal abgeblassten Papille ohne Stauungszeichen am linken Auge. Das Gesichtsfeld (GF) des betroffenen Auges zeigte eine Vergrößerung des blinden Flecks sowie einen diffusen Abfall in allen Quadranten. Die visuell evozierten Potentiale am LA waren sowohl in der Amplitude vermindert als auch in der Latenz erhöht. Der Farnsworth-Farbtest (15HUE) ergab eine unspezifische Alteration des Farbsehens am LA (RA: normal). Die sofort durchgeführte Kernspintomographie zeigte ein großes $(12 \times 14 \mathrm{~mm})$, nichtrupturiertes, partiell thrombosiertes, paraklinoidales Carotis-Ophthalmica-Aneurysma mit einer Kompression des N.opticus links. 
Abb. 1a, $b>$ Die Funduskopie ergab eine Tortuositas

vasorum bds. Das linke Auge zeigte eine temporal abgeblasste Papille ohne weitere Stauungszeichen
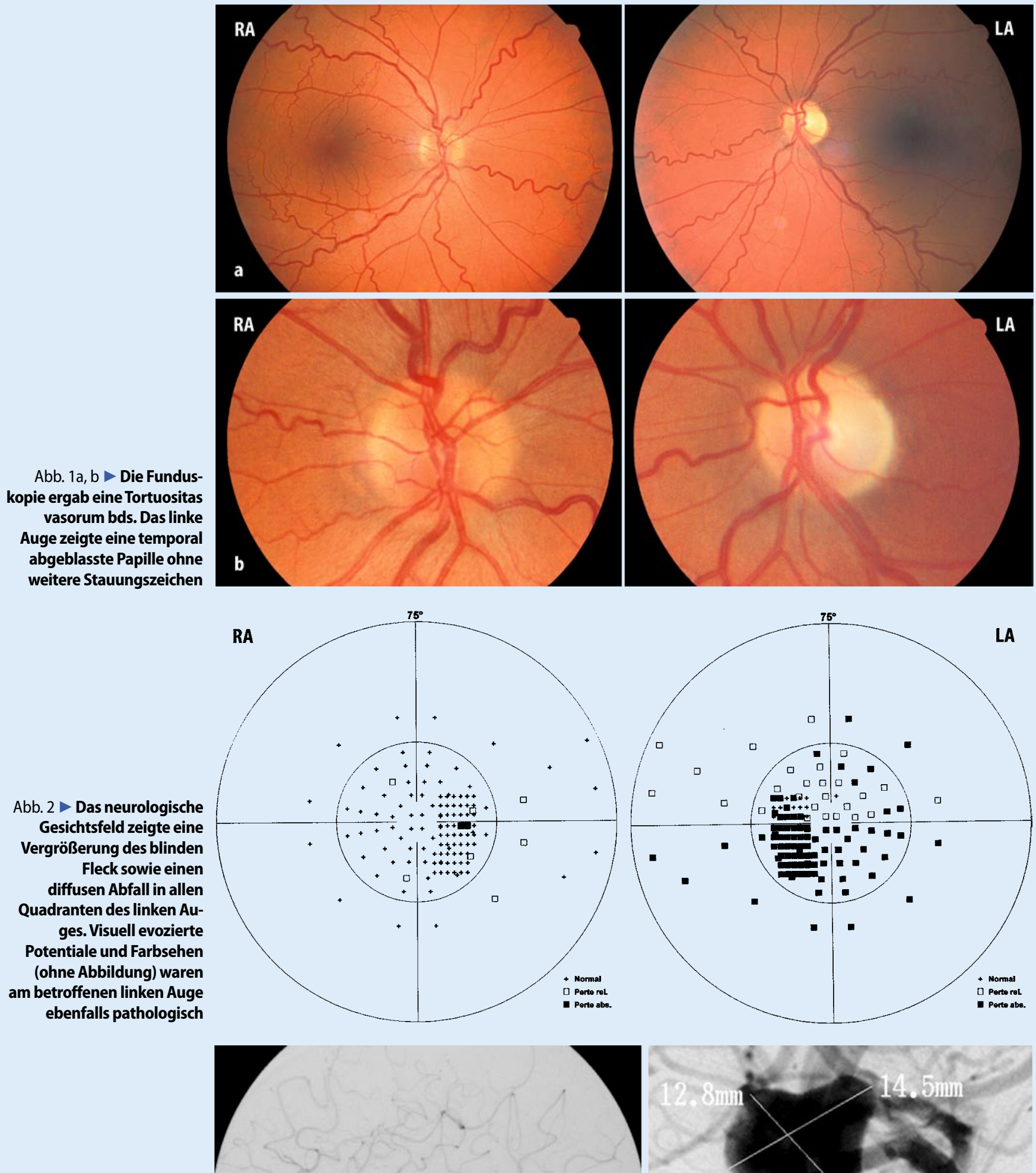

Abb. 3a, $b>$ Grosses (12x

$14 \mathrm{~mm}$ ), nichtrupturiertes, partiell thrombosiertes, paraklinoidales CarotisOphthalmica- Aneurysma mit Kompression des N. opticus links
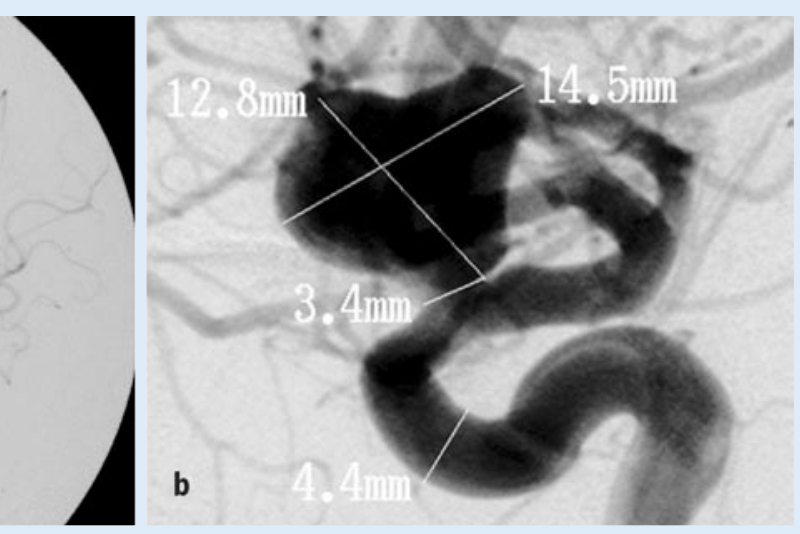

Ihre Diagnose? 


\section{\ Diagnose: Carotis-Ophthalmica- Aneurysma mit einer Kompression des optischen Sehnerven.}

\section{Therapie}

Der Patient wurde rasch einer osteoplastischen Kraniotomie links pterional und einem Aneurysma-Clipping mittels 2-Yasargil-Standart-Clips unterzogen.

Der weitere Heilungsverlauf gestaltete sich komplikationslos, auch der Visus und das GF zeigten eine baldige Verbesserung. Trotz der offensichtlichen teilweise vorhandenen Optikusatrophie mit geringen verbleibenden GF-Alterationen war der Visus der letzten Untersuchung 2 Jahre nach dem operativen Eingriff erfreulicherweise auf 10/10 s.c am LA angestiegen.

\section{Diskussion}

Eine Visusminderung kann Zeichen einer intrakraniellen vaskulären Malformation sein. Am häufigsten sind dabei intrakranielle Aneurysmen, Carotis-CavernosusFisteln sowie arteriovenöse und kavernöse Malformationen.

Die Prävalenz intrakranieller Aneurysmen liegt in großen Autopsieserien zwischen $1 \%$ und $6 \%$. In $20-30 \%$ dieser Patienten findet man multiple Aneurysmen [3]. Die Hauptlokalisationen liegen nach Schievink et al.[5] in über $80 \%$ im Bereich der anterioren Zirkulation, wobei Carotis-Ophthalmica-Aneurysmata zu den seltenen vaskulären Anomalien gehören [6].

Meist bleiben intrakranielle Aneurysmen bis zur Ruptur asymptomatisch. Lediglich 10\% zeigen Symptome, in erster Linie durch Verdrängung von umliegenden Strukturen („mass effect“, [1]). Neben uncharakteristischen Kopfschmerzen hängen die Symptome von der Lokalisation der Aneurysmata ab. Das neuroophthalmologische Hauptsymptom beim häufigsten intrakraniellen Aneurysma, das an der Verbindung zwischen der A. carotis interna und der A.communicans posterior liegt, ist eine Kompression des N. occulomotorius (III) im Subarachnoidal- raum. Seltener ist die Kompression von Hirnnerven im Sinus cavernosus, die sich über eine meist schmerzhafte einfache oder kombinierte Parese der okulären motorischen Nerven manifestiert. Ebenfalls seltener ist die direkte Kompression der optischen Nerven mit konsekutiver Visusminderung oder Gesichtsfeldausfällen. Aneurysmata, die sich über den "mass effect“ manifestieren, sind meist groß (>15 mm) und haben ein hohes Rupturrisiko von $6 \%$ im ersten Jahr. Dabei variiert das Intervall zwischen der Erstsymptomatik und der Aneurysmaruptur in der Regel zwischen einem Tag und 4 Monaten (median etwa 14 Tage). Eine rasche Diagnosestellung, wie in unserem Fallbeispiel dargestellt, kann daher einer massiven intrazerebralen oder subarachnoidalen Blutung vorbeugen.

Die jährliche Inzidenz von Rupturen intrakanieller Aneurysmen liegt bei o,5\%, die nachfolgenden intrazerebralen oder subarachnoidalen Blutungen dieser Rupturen weisen eine hohe Morbidität und Mortalität auf. 12\% der Patienten sterben bereits vor der ersten medizinischen Versorgung, 40\% innerhalb des ersten Monats nach der Ruptur. Mehr als 30\% der überlebenden Patienten weisen meist schwere neurologische Defekte auf [4].

Ophthalmologische Symptome rupturierter Aneurysmata reichen von Motilitätstörungen über eine Kompression der Sehbahn bis hin zu sekundären intraokularen Effekten der Subarachnoidalblutung (z. B. Terson-Syndrom, [2]).

\section{Korrespondierender Autor \\ Dr. R. Kiel}

Hôpital Jules Gonin,

Av. de France 15, 1004 Lausanne, Schweiz

E-Mail: kielralf@hotmail.com

Interessenkonflikt: Der korrespondierende Autor versichert, dass keine Verbindungen mit einer Firma, deren Produkt in dem Artikel genannt ist, oder einer Firma, die ein Konkurrenzprodukt vertreibt, bestehen.

\section{Literatur}

1. Barrow DL, Reisner A (1996) Natural history of intracranial aneurysms and vascular malformations. Clin Neurosurg 40:3-39

2. Biousse V, Mendicino ME, Simon DJ, Newman NJ (1998) The ophthalmology of intracranial vascular abnormalities. Am J Ophthalmol 125:527-544

3. Ingawa T, Hirano A (1990) Autopsy study of unruptured incidental intracranial aneurysms. Surg Neurol 34:361-365

4. Schievink WI, Wijdicks E, Parisi JE, Piepgras DG, Whisnant JP (1995) Sudden death from aneurysmal subarachnoidal hemorrhage. Neurology 45:871-874

5. Schievink WI (1997) Intracranial Aneurysms. N Engl J Med 336:28-40

6. Stroobandt G, Cornelis G (1971) Intrasellar carotidophtalmic aneurysm. Angiotomographic demonstration and surgical cure. Neurochirurgia 11:14 